\title{
Time-Averaged Conformations of the Inclusion Complexes of Cyclodextrin in Solution
}

\author{
Makoto KomiYAmA and Hidefumi HIRAI
}

Department of Industrial Chemistry, Faculty of Engineering, The University of Tokyo, 7-3-1 Bunkyo-ku, Hongo, Tokyo 113, Japan.

(Received July 25, 1980)

\author{
KEY WORDS Cyclodextrin / Inclusion Complex / Conformation / ${ }^{1} \mathrm{H}$ \\ Nuclear Magnetic Resonance Chemical Shift /
}

Cyclodextrins (CDs) are cyclic oligomers consisting of 6-8 $\alpha$-glucose units and have often been used as models of enzymes and as useful catalysts. ${ }^{1}$ This is mostly due to the formation of $\mathrm{CD}$-inclusion complexes with the substrates.

There is much evidence showing that guest compounds are really included in the cavities of CDs in solution. ${ }^{2-8}$ However, information on the positions of the guest compounds in the cavities in solution, which should be one of the factors governing the catalyses by $\mathrm{CDs}$, is still scarce. Furthermore, a direct inference of the structures of the inclusion complexes in solution from those in solid, determined by X-ray crystallography, ${ }^{9}$ is not always acceptable.

In this paper, the time-averaged positions of $p$ nitrophenolate (1), $p$-nitrophenol (2), benzoic acid (3), and benzoate (4) in the cavity of $\alpha$-cyclodextrin $(\alpha-C D)$ in an aqueous solution is determined by use of the changes in the ${ }^{1} \mathrm{H}$ nuclear magnetic resonance (NMR) chemical shifts of $\alpha-C D$ due to the anisotropic shielding effects from the aromatic rings of the guest compounds.

Changes in the ${ }^{1} \mathrm{H}$ NMR chemical shifts $\left(\Delta_{\mathrm{obs}}\right.$ 's) of the H-3 and H-5 protons of $\alpha-C D$ on the inclusion-complex formation were determined at $30^{\circ} \mathrm{C}$ using a JEOL PS- 100 spectrometer.

The magnitudes of the shielding effects $\left(\Delta_{\text {calc }}\right.$ 's) of the aromatic ring on the H-3 and H-5 protons of $\alpha$ $\mathrm{CD}$ were evaluated by using the table of Johnson and Bovery ${ }^{10}$ which shows the magnitudes of the shielding of the benzene ring on a proton as a function of the position of the proton with respect to the benzene ring. Calculation was made on the following assumptions.

1. The distances of the H-3 and H-5 atoms from the longitudinal axis ( $z$-axis) of the cavity of $\alpha$-CD are 3.5 and $2.9 \AA$, respectively, as shown in the study using the CPK (Corey-Pauling-Koltun) molecular model.

2. The center of the aromatic ring of the guest compound is on the $z$-axis.

3. The aromatic ring takes the two conformations A and B in Figure 1, the statistical weights of both of which are 0.5 . The third assumption is based on the ${ }^{1} \mathrm{H}$ NMR study on the inclusion complexes of $\alpha-C D$ with $1-4$, showing only the time-averaged signal for each of the protons of $\alpha$ CD. The $\Delta_{\text {calc }}$ of the H-3 or H-5 atoms was obtained as a function of the height of the center of the aromatic ring with respect to the plane comprised of the six $\mathrm{H}-3$ or $\mathrm{H}-5$ atoms, by averaging the twelve values of the shielding effects on the corresponding protons. Six of the twelve values refer to the six glucose units of $\alpha-C D$ in the conformation $A$, whereas the rest correspond to those in the

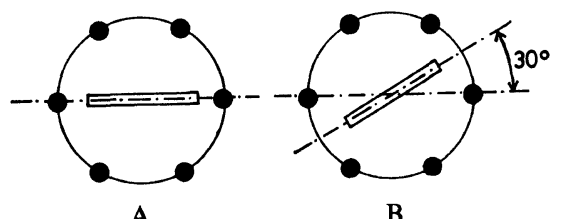

Figure 1. Conformations $\mathrm{A}$ and $\mathrm{B}$ used for calculating $\Delta_{\text {calc }}$ 's. The black circles and the rectangles, respectively, refer to the positions of the H-3 or H-5 atoms of $\alpha-C D$ and the orientation of the plane of the aromatic ring of the guest compound. 


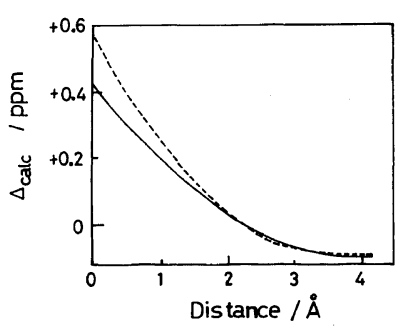

Figure 2. Plots of the magnitudes of the shielding effects of an aromatic ring $\left(\Delta_{\text {calc }}\right)$ on the $\mathrm{H}-3$ atoms (the solid line) and the H-5 atoms (the dotted line) vs. the distance between the center of the aromatic ring and the plane comprised of the six of the corresponding hydrogen atoms. The positive sign shows increase in the shielding.

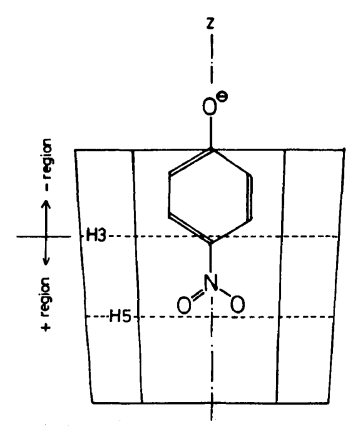

A

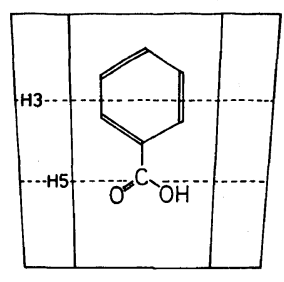

B
Figure 3. Time-averaged conformations of the $\alpha-C D-1$ complex (A) and the $\alpha$-CD-3 complex (B). ---H3--- and ---H5--- show the planes comprised of the six H-3 and $\mathrm{H}-5$ atoms, respectively.

conformation B. The optimal position was determined by comparing $\Delta_{\text {calc }}$ 's with $\Delta_{\text {obs }}$ 's at various positions of the aromatic ring of the guest compound. At the optimal position, $\Delta_{\text {calc }}$ 's show maximal agreements with $\Delta_{\mathrm{obs}}$ 's for the H-3 and H-5 atoms of $\alpha-\mathrm{CD}$. This position was taken as the timeaveraged position. Analyses on the protons of $\alpha$-CD other than the $\mathrm{H}-3$ and $\mathrm{H}-5$ ones were not attempted, since they were so far away from the guest compounds that the table in ref 10 was not applicable. The shielding effects of the substituent groups of the benzene rings of the guest compounds were not considered in the calculation of $\Delta_{\text {calc }}$, since the magnitudes were minor compared with those of the effects of the aromatic rings. ${ }^{11}$

Figure 2 shows the dependence of $\Delta_{\text {calc }}$ on the distance between the center of the aromatic ring and
Table I. Values of $\Delta_{\text {obs }}$ and $\Delta_{\text {calc }}$ for the complex formation of $\alpha$-cyclodextrin with $p$-nitrophenolate, $p$-nitrophenol, benzoic acid, and benzoate ${ }^{\mathrm{a}}$

\begin{tabular}{|c|c|c|c|}
\hline \multirow{2}{*}{$\begin{array}{l}\text { Guest } \\
\text { compound }\end{array}$} & \multirow{2}{*}{$\begin{array}{c}\text { Proton } \\
\text { of } \\
\alpha \text {-cyclodextrin }\end{array}$} & \multirow{2}{*}{$\frac{\Delta_{\text {obs }}}{\mathrm{ppm}}$} & \multirow{2}{*}{$\frac{\Delta_{\text {calc }}}{\mathrm{ppm}}$} \\
\hline & & & \\
\hline \multirow{2}{*}{$p$-Nitrophenolate } & $\mathrm{H}-3$ & +0.20 & +0.21 \\
\hline & $\mathrm{H}-5$ & -0.05 & -0.08 \\
\hline \multirow{2}{*}{$p$-Nitrophenol } & $\mathrm{H}-3$ & +0.25 & +0.26 \\
\hline & $\mathrm{H}-5$ & -0.04 & -0.08 \\
\hline \multirow{2}{*}{ Benzoic acid } & $\mathrm{H}-3$ & +0.41 & +0.40 \\
\hline & $\mathrm{H}-5$ & -0.08 & -0.09 \\
\hline \multirow{2}{*}{ Benzoate } & $\mathrm{H}-3$ & +0.30 & +0.30 \\
\hline & $\mathrm{H}-5$ & +0.01 & +0.02 \\
\hline
\end{tabular}

a The positive sign refers to the change in the ${ }^{1} \mathrm{H}$ NMR chemical shift toward a higher magnetic field with respect to the chemical shift in the absence of the guest compound.

the plane comprised of all the H-3 or H-5 atoms of the six glucose units of $\alpha-C D$.

By using the results in Figure 2 and $\Delta_{\text {obs }}$ 's, the time-averaged positions of the aromatic rings of 14 in the $\alpha-C D$ inclusion complexes in aqueous solution were estimated. The heights of the centers of the aromatic rings of $1,2,3$, and 4 with respect to the plane comprised of the six $\mathrm{H}-3$ atoms were determined to be $-1.1,-0.7,-0.1$, and $+0.5 \AA$, respectively. Here, the negative sign refers to the region in the side of the secondary hydroxyl groups of $\alpha$-CD (see Figure 3). At these positions, the $\Delta_{\text {calc }}$ 's for both H-3 and H-5 atoms agree fairly well with the corresponding $\Delta_{\text {obs }}$ 's, (Table I).

Figure 3 depicts the time-averaged conformations of the $\alpha-C D-1$ and the $\alpha-C D-2$ complexes. The positions of the functional groups, that is, the directions of the penetrations of the guest compounds were determined according to the literature. ${ }^{3,12}$

The validity of the present procedure for determining the time-averaged conformations of the inclusion complexes was clearly shown by the results of the ${ }^{1} \mathrm{H}$-homonuclear Overhauser effects in the $\alpha$ CD-1 complex. ${ }^{13}$. The irradiation of the resonance of the H-3 atoms showed a much larger enhance- 
ment of the intensities of the signals of the metaprotons of 1 than the ortho-protons. The conformation of the $\alpha-C D-1$ complex shown in Figure 3, where the $\mathrm{H}-3$ atoms and the meta-protons are in a close proximity, is satisfactorily consistent with these results.

Furthermore, the deeper penetration of the aromatic ring of 3 to the cavity of $\alpha-C D$ than the penetration of the aromatic ring of 1 indicated in this study is consistent with the ${ }^{13} \mathrm{C}$ NMR spectroscopy, showing that the change in the chemical shift toward the lower magnetic field of the ortho-carbon of 3 due to the complex formation with $\alpha$-CD (1.67 $\mathrm{ppm})$ is larger than the corresponding value of the meta-carbon of $1(1.15 \mathrm{ppm})$.

In conclusion, the time-averaged positions of $p$ nitrophenolate, $p$-nitrophenol, benzoic acid, and benzoate in the cavity of $\alpha$-cyclodextrin in aqueous solution were determined using ${ }^{1} \mathrm{H}$ NMR spectroscopy.

Acknowledgment. The present work was partially supported by a Grant-in-Aid for Scientific Research from the Ministry of Educations, Science and Culture.

\section{REFERENCES}

1. M. L. Bender and M. Komiyama, "Cyclodextrin Chemistry," Springer-Verlag, Berlin, 1978.

2. D. J. Wood, F. E. Hruska, and W. S̀aenger, J. Am. Chem. Soc., 99, 1735 (1977).

3. R. Bergeron, M. A. Channing, and K. A. McGovern, J. Am. Chem. Soc., 100, 2878 (1978).

4. P. V. Demarco and A. L. Thakkar, Chem. Commun., 2 (1970).

5. A. L. Thakkar and P. V. Demarco, J. Pharm. Sci., 60, 652 (1971).

6. Y. Inoue, Y. Katôno, and R. Chûjô, Bull. Chem. Soc. Jpn., 52, 1692 (1979).

7. J. P. Behr and J. M. Lehn, J. Am. Chem. Soc., 98, 1743 (1976).

8. K. Uekama, F. Hirayama, N. Matsuo, and H. Koinuma, Chem. Lett., 703 (1978).

9. K. Harata, Bull. Chem. Soc. Jpn., 52, 2451 (1979) and the references cited therein.

10. C. E. Johnson and F. A. Bovey, J. Chem. Phys., 29, 1012 (1958). The table shown in the following book was used here; J. W. Emsley, J. Feeney, and L. H. Sutcliffe, "High Resolution Nuclear Magnetic Resonance Spectroscopy, Vol. I," Pergamon Press, Oxford, 1965, Appendix B, p 595.

11. L. M. Jackman and S. Sternhell, "Application of Nuclear Magnetic Resonance Spectroscopy in Organic Chemistry," Pergamon Press, Oxford 1969.

12. R. J. Bergeron, M. A. Channing, G. J. Gibeily, and D. M. Pillor, J. Am. Chem. Soc., 99, 5146 (1977).

13. R. J. Bergeron and R. Rowan, III, Bioorg. Chem., 5, 425 (1976). 